\title{
Practice Note: 'If Only All Women Menstruated Exactly Two Weeks Ago': Interdisciplinary Challenges and Experiences of Capturing Hormonal Variation Across the Menstrual Cycle
}

\author{
Lauren C. Houghton and Noémie Elhadad
}

Menstrual health is important in its own right, but menstrual characteristics, including the signs and symptoms that accompany the cycle and their underlying hormonal patterns, are also risk factors for chronic diseases such as breast cancer and endometriosis, two diseases to which we have dedicated our own research careers. One such risk factor for both diseases is early age at menarche (Bodicoat et al. 2014; Parazzini et al. 2017). When one of us got her first period at age 12.11 years ( $\mathrm{LCH}$ knows her exact age because it was on Christmas Eve), she was ashamed. Now 24 years and 288 menstrual cycles later, she has personally interviewed over 1000 girls and women about their menstrual cycles and the shame has subsided (Houghton et al. $2014,2018,2019)$. When the other got her first period at age 13.9 years, she soon realized that periods were going to play a painful part of her life. She was later diagnosed with endometriosis and has since designed technology to give a voice to women living with endometriosis (McKillop, Mamykina, and Elhadad 2018; Urteaga et al. 2018). Since their first periods, secular changes in societal views have also been dramatic: Teenage girls are coding their own video games "to rid the world of the menstrual taboo" "Tampon Run," n.d.), governments are passing "Menstrual Equity" legislation (Zraick 2018), and women downloading period tracking apps have made them the second most used health app (PRIORI DATA). These societal and technological changes have also changed the way we and other anthropologists, 
epidemiologists, and data scientists interested in characterizing hormonal variation in menstrual patterns conduct menstruation research.

Anthropologists have been instrumental in demonstrating that there is global variation in menstruation, its timing across the life course, the cultural practices surrounding it, and the underlying hormones that regulate it. Starting in the 1980s, biological anthropologists went to the field in various populations and compared menstrual cycles among women living in vastly different ecological contexts with starkly different norms surrounding reproductive timing. They demonstrated that the Dogon women of West Africa menstruate three times less than American and European women in their lifetime (Strassmann 1999). This dramatic difference in lifetime number of menstrual cycles is explained by the later age at menarche, earlier age at first pregnancy, higher parity, and earlier age at menopause in the Dogon women.

The variation in number of lifetime menstrual cycles is also reflected in underlying variation in reproductive hormones. For example, the overall pattern of progesterone across the menstrual cycle is similar among American, Polish, Lese, and Nepali women, with levels low and flat during the follicular phase (days 1-14), rising during ovulation (day 14), peaking during the mid-luteal phase (day 21), and slowly declining in the late luteal phase until day 28. However, the absolute levels of hormone at each of these phases is three-fold higher in American and Polish women compared to the Lese and Nepalese (Ellison 1994). The variation in hormone profiles demonstrates natural variation in ovarian function across populations, and this variation should be considered normal rather than pathological (Ellison et al. 1993).

Technological advances in hormone measurement made this important research possible. Peter Ellison and his colleagues leveraged the advancement in assays (Riad-Fahmy et al. 1982) to measure hormones in saliva for field settings (Ellison 1993). The advantage of using saliva was that anthropologists could collect daily samples from women and, using preservatives, store the samples at room temperature in isolated field locations where refrigeration was not possible (Lipson and Ellison 1989). It was important to collect daily samples to capture the hormonal profile of the entire cycle and to detect day of ovulation in order to align cycles.

Epidemiologists have also been interested in the variation of menstrual patterns (timing and frequency) and reproductive hormones because of their associations with breast cancer (Mumford et al. 2012; Whelan et al. 1994; Terry et al. 2005). As a result, hundreds of studies have compared hormones between women with and without breast cancer; between women with different risk factors, such as reproductive events, diets, and physical activity levels; and between women living in populations with different breast cancer incidence rates. Epidemiologists, who typically work with much larger study populations than anthropologists, conducted these comparisons using serum or urine samples collected on specific days of the menstrual cycle. In order to correctly time collection, epidemiologists asked women to recall the 
date of their last menstrual period (LMP) and then count forward to schedule a specimen collection on the targeted day. In most cases the target day would be menstrual cycle day 21 , when both progesterone and estradiol are relatively high.

Restricting comparison of hormones to specific days is cost-effective for large-scale studies, but it comes at a scientific cost in studies of premenopausal women. Characterizing a woman's "dose" of estrogen based on only one or two timed samples may not capture the full variation in hormones over the menstrual cycle. For example, we know elevated estrogen levels are associated with increased risk for breast cancer among postmenopausal women (from whom a single sample can be collected since there is no menstrual cycle variation to account for), but we are less confident this association holds in premenopausal women (Key 2011). Additionally, follicular phase estradiol is associated with increased breast cancer risk, whereas luteal phase estradiol is not (Eliassen et al. 2006). These gaps in understanding regarding premenopausal hormones and their relationship with breast cancer are particularly problematic given that breast cancer incidence is on the rise, specifically in US women under 40 years old (Johnson, Chien, and Bleyer 2013). So while epidemiologists have also contributed to knowledge through large cohort studies, their narrow focus on measuring hormone levels on specific days has limited our understanding of the full variability of hormones in menstruation.

Further, calibrating the hormone samples to the cycle day relies on women's ability to recall the date of their LMP, but cycle lengths vary within and between women and women's recollections may not be accurate. For example, some epidemiologists have followed up with women to confirm that the sample collection in fact occurred on or near the targeted day. They provided participants, who in this case were highly motivated nurses, with a pre-addressed postcard to return with the date when their next period began. They found that specimens were collected as many as four days before or after the target day in the best-case scenarios (Eliassen et al. 2006).

In our experience, some women record their menstrual period in their own personal calendar, but many women do not. Over the course of interviewing women and asking them to recall their LMP, we have observed several estimation techniques. If her period just finished, she would count back five days to the day it began. Or, she would think of where she was the last time it occurred and then work out the date based on where she was that month. If her period had not been within the last week or if there was no significant event to help jog her memory, the most common response was to default to "oh, about two weeks ago." This suggests that self-reported LMP is a rather rough estimate.

Advancements in statistical methods can overcome some of these challenges when collecting daily specimens is not feasible. In one study, one of us took an alternative approach to targeting a specific cycle day. We collected specimens from all of the women in our study over the same two calendar days and later asked them to confirm when their next menstrual period 
(NMP) started (Houghton et al. 2016; Troisi et al. 2014). Thus, by chance, women were at different days of their menstrual cycle and this yielded a collection of samples randomly distributed across a menstrual cycle. We then took this random distribution and modeled (using cubic splines) the hormone profile at the population level using individual data on specific days. Without giving the model a specified shape, the curves mimicked biological curves seen in individual women. We were then able to compare hormone concentrations between populations by calculating the area under each curve. In this specific case, we were able to demonstrate that estrogens were actually higher in Mongolian women than in British women, a finding that was counter to what most people would predict, given that Mongolia has some of the lowest breast cancer rates in the world (Troisi et al. 2014). Confirming the date of the NMP was of the utmost importance, to make sure we knew the exact menstrual day on which the sample had been collected. Data scientists use even more sophisticated modeling and prediction techniques to predict the hormone profiles underlying women's menstrual cycle characteristics. For example, the state-of-the-art model developed by Clark and colleagues accurately captures the same hormonal patterns as a dataset of daily hormonal measurements from healthy women (Harris Clark, Schlosser, and Selgrade 2003).

Our own experience in collecting and analyzing menstrual cycle and hormonal data mirrors the methodologies in the literature, ranging from small-scale studies with "in-depth" hormone measurements (daily readings throughout the menstrual cycle) to large-scale cohort analysis with "shallow" hormone measurements (under-sampling in time, or sampling only at known phases of the cycle). The "deepest" examples can be found in reproductive endocrinology, where researchers have established basic knowledge about the cycle through small-scale studies of 12 women and 15-minute interval blood serum measurements (Murdoch et al. 1985). But such a deep dive is not the route we have chosen to follow. Rather, we are both now excited to incorporate mobile apps into our research toolbox to mitigate the tension between the need for multiple measurements and the invasive nature of taking such measurements. Mobile health is a game changer for investigating the menstrual cycle, making data collection possible at an unprecedented scale. Women can now use menstrual tracking apps to track their own cycles, along with a range of signs and symptoms - data which previously did not get recorded. It is not only possible, women are doing it. Cycle tracking apps are some of the fastest-growing health apps and have a loyal base of users (Fox and Duggan 2012; Wartella et al. 2016). In 2018, 48\% of US females ages 18 - to 22 -years-old and $25 \%$ of teen girls reported having used a period tracking app (Fox 2018). Some apps have as many as 10 million users worldwide ("Clue Wants Your Research Proposals on Reproductive Health," n.d.). There is a great opportunity to leverage self-tracking technology to enable characterizing the menstrual cycle at scale and for individuals. 
One promising aspect of apps used for period tracking is their flexibility in capturing a range of experiences related to menstruation. Popular trackers enable users to self-track their cycle and period lengths, as well as specific symptoms (such as, headaches, sleep, bowel movements, pain levels); psychosocial elements (such as, moods or socializing patterns); and sexual activity. Many apps further enable users to customize their tracking options, thus getting closer to capturing the broader context and the narrative around menstruation. While cycle characteristics on their own might not be enough for algorithms to model cycle variations, the additional data elements can significantly boost these models' ability to infer underlying hormonal variations.

Beyond self-tracked data, emerging analytics can help us determine the ideal hormone-sampling rate for future studies or improved diagnostics. For example, Urteaga and colleagues $(2017,2019)$ showed that machine learning-based approaches that combine the aforementioned state-of-the art model for daily average hormone concentrations with cycle data collected using a mobile phone application can accurately identify the phases within the menstrual cycle, even without daily measurements. Furthermore, the model allows for generation of hormone patterns with different characteristics, such as varying cycle length. Once these methods are validated with in-depth hormonal datasets, they have the potential to revolutionize how we study the variation in menstrual cycle and hormonal patterns in healthy and unhealthy women.

As app developers try to perfect their algorithms to predict each user's next period more accurately, and researchers try to use app tracking data to predict underlying hormonal profiles, this momentum must be accompanied by some caution and critical reflections. First, there is the risk that only using data collected by menstruators who use apps may further marginalize those menstruators who don't use apps for biological (irregular periods) or socially patterned reasons. For example, while smart phone and app use is global, within emerging markets people with less education are still less likely to use apps than those with more education (Taylor and Silver 2019). Second, new mHealth data collection methods may lead to large menstrual cycles studies at unprecedented scales, but the cultural norms with which the technology was developed may obscure the cultural context of the populations under study (Fox and Epstein [Chapter 54] in this volume). Principles of citizen science might be helpful in engaging on-the-ground perspectives from diverse groups of menstruators. From the collective work of anthropologists, epidemiologists, and data scientists, we know that our culture and biology both influence our menstrual health. We should ensure that our methods are equally attuned to biological plausibility and cultural context. 


\section{REFERENCES}

Bodicoat, Danielle H., Minouk J. Schoemaker, Michael E. Jones, Emily McFadden, James Griffin, Alan Ashworth, and Anthony J. Swerdlow. 2014. "Timing of Pubertal Stages and Breast Cancer Risk: The Breakthrough Generations Study." Breast Cancer Research 16 (1): R18. https://doi.org/10.1186/bcr3613.

"Clue Wants Your Research Proposals on Reproductive Health." n.d. Accessed March 19, 2019. https://helloclue.com/articles/about-clue/clue-research-innovationprogram-call-for-proposals.

Eliassen, A. Heather, Stacey A. Missmer, Shelley S. Tworoger, Donna Spiegelman, Robert L. Barbieri, Mitch Dowsett, and Susan E. Hankinson. 2006. "Endogenous Steroid Hormone Concentrations and Risk of Breast Cancer among Premenopausal Women." Journal of the National Cancer Institute 98 (19): 1406-15. https://doi. org/10.1093/jnci/djj376.

Ellison, P. T. 1993. "Measurements of Salivary Progesterone." Annals of the New York Academy of Sciences 694 (September): 161-76. http://www.ncbi.nlm.nih.gov/ pubmed/8215052.

- 1994. "Salivary Steroids and Natural Variation in Human Ovarian Function." Annals of the New York Academy of Sciences 709 (February): 287-98. http://www. ncbi.nlm.nih.gov/pubmed/8154721.

Ellison, P. T., C. Panter-Brick, S. F. Lipson, and M. T. O'Rourke. 1993. "The Ecological Context of Human Ovarian Function." Human Reproduction 8 (12): 2248-58. http://www.ncbi.nlm.nih.gov/pubmed/8150934.

Fox, Susannah. 2018. "Digital Health Practices among Teens and Young Adults in the U.S.” 2018. https://susannahfox.com/2018/07/31/digital-health-practicesamong-teens-and-young-adults-in-the-u-s/.

Fox, S., and M. Duggan. 2012. "Mobile Health 2012 | Pew Research Center." n.d. Accessed March 19, 2019. http://www.pewinternet.org/2012/11/08/ mobile-health-2012/.

Harris Clark, L., Paul M. Schlosser, and James F. Selgrade. 2003. "Multiple Stable Periodic Solutions in a Model for Hormonal Control of the Menstrual Cycle." Bulletin of Mathematical Biology 65 (1): 157-73. https://doi.org/10.1006/ bulm.2002.0326.

Houghton, L. C., G. D. Cooper, G. R. Bentley, M. Booth, O. A. Chowdhury, R. Troisi, R. G. Ziegler, R. N. Hoover, and H. A. Katki. 2014. "A Migrant Study of Pubertal Timing and Tempo in British-Bangladeshi Girls at Varying Risk for Breast Cancer.” Breast Cancer Research 16 (1). https://doi.org/10.1186/ s13058-014-0469-8.

Houghton, Lauren, Davaasambuu Ganmaa, Philip Rosenberg, Dambadarjaa Davaalkham, Frank Stanczyk, Robert Hoover, and Rebecca Troisi. 2016. "Associations of Breast Cancer Risk Factors with Premenopausal Sex Hormones in Women with Very Low Breast Cancer Risk." International Journal of Environmental Research and Public Health 13 (12): 1066. https://doi. org/10.3390/ijerph13111066.

Houghton, L. C., J. A. Knight, M. J. De Souza, M. Goldberg, M. L. White, K. O’Toole, W. K. Chung, et al. 2018. “Comparison of Methods to Assess Onset of Breast Development in the LEGACY Girls Study: Methodological Considerations for Studies of Breast Cancer." Breast Cancer Research 20 (1). https://doi. org/10.1186/s13058-018-0943-9. 
Houghton, Lauren C., Julia A. Knight, Ying Wei, Russell D. Romeo, Mandy Goldberg, Irene L. Andrulis, Angela R. Bradbury, et al. 2019. "Association of Prepubertal and Adolescent Androgen Concentrations with Timing of Breast Development and Family History of Breast Cancer." JAMA Network Open 2 (2): e190083. https://doi.org/10.1001/jamanetworkopen.2019.0083.Johnson.

Johnson, Rebecca H., Franklin L. Chien, and Archie Bleyer. 2013. "Incidence of Breast Cancer with Distant Involvement among Women in the United States, 1976 to 2009." JAMA 309 (8): 800-805. https://doi.org/10.1001/jama.2013.776.

Key, Timothy J. 2011. "Endogenous Oestrogens and Breast Cancer Risk in Premenopausal and Postmenopausal Women.” Steroids 76 (8): 812-15. https:// doi.org/10.1016/J.STEROIDS.2011.02.029.

Lipson, S. F., and P. T. Ellison. 1989. "Development of Protocols for the Application of Salivary Steroid Analysis to Field Conditions." American Journal of Human Biology 1 (3): 249-55. https://doi.org/10.1002/ajhb.1310010304.

McKillop, Mollie, Lena Mamykina, and Noémie Elhadad. 2018. "Designing in the Dark." In Proceedings of the 2018 CHI Conference on Human Factors in Computing Systems-CHI '18, 1-15. ACM Press, New York, New York, USA. https://doi. org/10.1145/3173574.3174139.

Mumford, Sunni L., Anne Z. Steiner, Anna Z. Pollack, Neil J. Perkins, Amanda C. Filiberto, Paul S. Albert, Donald R. Mattison, Jean Wactawski-Wende, and Enrique F. Schisterman. 2012. "The Utility of Menstrual Cycle Length as an Indicator of Cumulative Hormonal Exposure." The Journal of Clinical Endocrinology of Metabolism 97 (10): E1871-79. https://doi.org/10.1210/jc.2012-1350.

Murdoch, A. P., P. J. Diggle, W. Dunlop, and P. Kendall-Taylor. 1985. "Determination of the Frequency of Pulsatile Luteinizing Hormone Secretion by Time Series Analysis.” Clinical Endocrinology 22 (3): 341-46. http://www.ncbi. nlm.nih.gov/pubmed/3978835.

Parazzini, F., G. Esposito, L. Tozzi, S. Noli, and S. Bianchi. 2017. "Epidemiology of Endometriosis and Its Comorbidities." European Journal of Obstetrics \& Gynecology and Reproductive Biology 209 (February): 3-7. https://doi.org/10.1016/j. ejogrb.2016.04.021.

“PRIORI DATA-Mobile App Data Intelligence, ASO Tool \& Market Research.” n.d.

Riad-Fahmy, D., G. F. Read, R. F. Walker, and K. Griffiths. 1982. "Steroids in Saliva for Assessing Endocrine Function.” Endocrine Reviews 3 (4): 367-95. https://doi. org/10.1210/edrv-3-4-367.

Strassmann, B. I. 1999. "Menstrual Cycling and Breast Cancer: An Evolutionary Perspective." Journal of Women's Health 8 (2): 193-202. http://www.ncbi.nlm. nih.gov/pubmed/10100133.

“Tampon Run.” n.d. Accessed March 19, 2019. http://tamponrun.com/.

Taylor, Ken, and Laura Silver. 2019. "Smartphone Ownership Is Growing Rapidly Around the World, but Not Always Equally | Pew Research Center." Pew Research Center, 2019. http://www.pewglobal.org/2019/02/05/smartphone-ownershipis-growing-rapidly-around-the-world-but-not-always-equally/.

Terry, Kathryn L., Walter C. Willett, Janet W. Rich-Edwards, David J. Hunter, and Karin B. Michels. 2005. "Menstrual Cycle Characteristics and Incidence of Premenopausal Breast Cancer." Cancer Epidemiology Biomarkers \& Prevention 14 (6): 1509-13. https://doi.org/10.1158/1055-9965.EPI-05-0051. 
Troisi, Rebecca, Daavasambuu Ganmaa, Isabel dos Santos Silva, Dambadarjaa Davaalkham, Philip S. Rosenberg, Janet Rich-Edwards, Lindsay Frasier, et al. 2014. "The Role of Hormones in the Differences in the Incidence of Breast Cancer between Mongolia and the United Kingdom." PLoS One 9 (12): el14455. https://doi.org/10.1371/journal.pone.0114455.

Urteaga, Iñigo, David J. Albers, Marija Vlajic Wheeler, Anna Druet, Hans Raffauf, and Noémie Elhadad. 2017. "Towards Personalized Modeling of the Female Hormonal Cycle: Experiments with Mechanistic Models and Gaussian Processes," Machine Learning for Health Workshop at NIPS (ML4H'17).

Urteaga, Iñigo, Mollie McKillop, Sharon Lipsky-Gorman, and Noémie Elhadad. 2018. "Phenotyping Endometriosis through Mixed Membership Models of Self-Tracking Data," November. Machine Learning for Healthcare (MLHC'18).

Urteaga, Iñigo, Tristan Bertin, Theresa Hardy, David Albers, and Noémie Elhadad. 2019. "Multi-Task Gaussian Processes and Dilated Convolutional Networks for Reconstruction of Reproductive Hormonal Dynamics," Machine Learning for Healthcare (MLHC'19).

Wartella, Ellen, Vicky Rideout, Heather Montague, Leanne Beaudoin-Ryan, and Alexis Lauricella. 2016. "Teens, Health and Technology: A National Survey." Media and Communication 4 (3): 13. https://doi.org/10.17645/mac.v4i3.515.

Whelan, E. A., D. P. Sandler, J. L. Root, K. R. Smith, and C. R. Weinberg. 1994. "Menstrual Cycle Patterns and Risk of Breast Cancer." American Journal of Epidemiology 140 (12): 1081-90. http://www.ncbi.nlm.nih.gov/ pubmed/7998590.

Zraick, Karen. 2018. "It's Not Just the Tampon Tax: Why Periods Are Political." The New York Times, July 22, 2018. https://www.nytimes.com/2018/07/22/health/ tampon-tax-periods-menstruation-nyt.html.

Open Access This chapter is licensed under the terms of the Creative Commons Attribution 4.0 International License (http://creativecommons.org/licenses/ by $/ 4.0 /$ ), which permits use, sharing, adaptation, distribution and reproduction in any medium or format, as long as you give appropriate credit to the original author(s) and the source, provide a link to the Creative Commons license and indicate if changes were made.

The images or other third party material in this chapter are included in the chapter's Creative Commons license, unless indicated otherwise in a credit line to the material. If material is not included in the chapter's Creative Commons license and your intended use is not permitted by statutory regulation or exceeds the permitted use, you will need to obtain permission directly from the copyright holder.

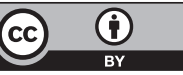

